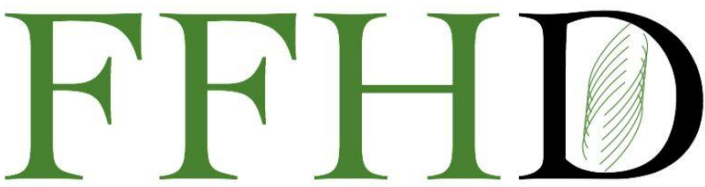

Functional Foods in Health and Disease

\title{
Serum levels of vitamin D, calcium, phosphorus, and oxidative parameters in healthy and diabetic people
}

\begin{abstract}
Maryam Barghi ${ }^{1}$, Amir Sadeghipoor Ranjbar ${ }^{2}$, Homa Moazen ${ }^{3}$, Narges Eskandari-Roozbahani ${ }^{*}$
${ }^{1}$ Department of Biochemistry, Faculty of Veterinary Medicine, Shiraz University, Shiraz, Iran; ${ }^{2}$ Department of Microbiology, Faculty of Engineering, Islamic Azad University, Sirjan Branch, Kerman, Iran; ${ }^{3}$ Department of Biostatics and Epidemiology, Shahid Sadoughi University of Medical Science, Yazd, Iran; ${ }^{4}$ Clinical Research Development Center, Imam Reza Hospital, Kermanshah University of Medical Sciences, Kermanshah, Iran
\end{abstract}

*Corresponding Author: Dr. Narges Eskandari Roozbahani, Clinical Research Development Center, Imam Reza Hospital, Kermanshah University of Medical Sciences, Kermanshah, Iran

Submission Date: March 11 ${ }^{\text {th }}, 2021$; Acceptance Date: May 13 ${ }^{\text {th }}, 2021$; Publication Date: May 19 ${ }^{\text {th }}, 2021$

Please cite this article as: Barghi M., Ranjbar A.S., Moazen H., Eskandari-Roozbahani N. Serum levels of vitamin D, calcium, phosphorus, and oxidative parameters in healthy and diabetic people. Functional Foods in Health and Disease 2021; 11(5): 238-245. DOI: https://www.doi.org/10.31989/ffhd.v11i5.787

\section{ABSTRACT}

Introduction: Diabetes mellitus is a metabolic disease that is a primary public health consideration. Low Vitamin D levels are linked to type 2 diabetes (T2DM), diminished insulin release, and enhanced insulin resistance in humans and animals. Vitamin D is also involved in the regulation of calcium and phosphorus homeostasis. Oxidative stress and antioxidant imbalances are important for the progression of diabetes as well. In this endeavor, the levels of vitamin D, calcium, phosphorus, and evaluation of the oxidantantioxidant factors of malondialdehyde (MDA) and total antioxidant capacity (TAC) in healthy and diabetic people were compared.

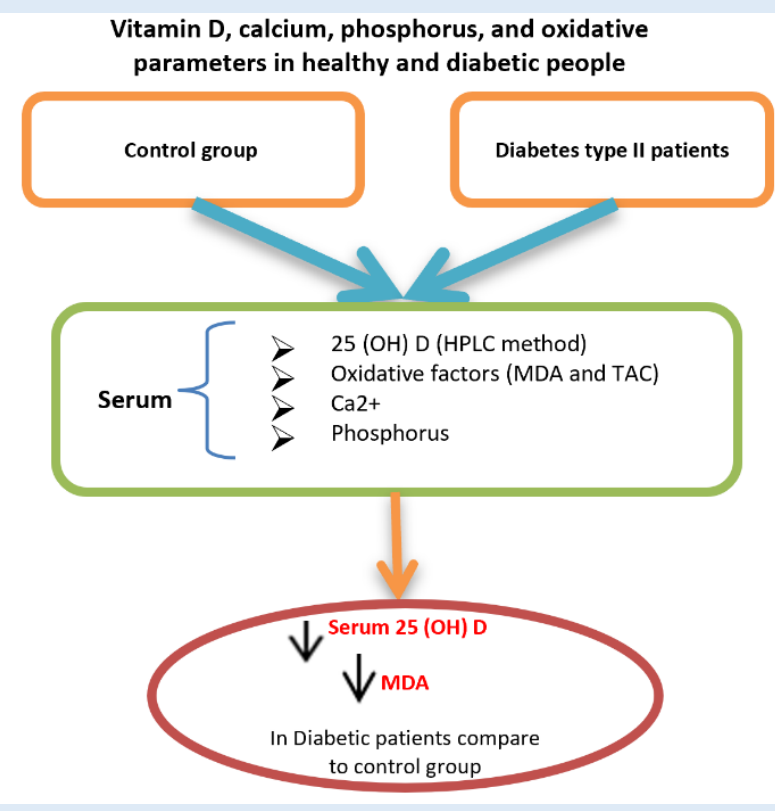

Methods: This descriptive-analytical study was conducted in 2020 in Shiraz, Fars province, Iran. The population included 40 T2DM patients (with HbA1c equivalent 6-8) without comorbidities, 20-60 years old for both genders, 
and 40 healthy individuals (female and male between 20-60 years old without comorbidities). The highperformance liquid chromatography (HPLC) method was adopted for measuring Vitamin D and for measuring other levels, the colorimetric method was used. Using SPSS 22, statistical analysis was performed. The MannWhitney $U$ test for quantitative data was applied. $P<0.05$ was deemed significant.

Results: There was a statistically significant difference between the two groups when it came to the means of vitamin D and MDA. In the diabetic group, vitamin D levels were lower $(p=0.001)$ and MDA levels were higher $(p<0.001)$. Comparing the level of calcium and phosphorus in diabetics and healthy people revealed no significant difference. This result was also true for the TAC test.

Conclusions: According to our results, the mean of vitamin D in T2DM was significantly lower than healthy people and MDA in T2DM significantly increased compared to the control group, suggesting that increasing the activity of this enzyme in the development of secondary complications in diabetic patients is a predisposing factor.

Keywords: Vitamin D, Diabetes mellitus, HPLC, Oxidative stress

CFFC 2021. This is an Open Access article distributed under the terms of the Creative Commons Attribution 4.0 License (http://creativecommons.org/licenses/by/4.0)

\section{INTRODUCTION}

Type 2 diabetes mellitus (T2DM) is characterized by a chronic rise in blood sugar due to a relative lack of insulin, insulin resistance, or both. Diabetes and its late effects lead to reduced life expectancy and health costs [1]. T2DM has become one of the most momentous and common health problems in recent decades and is now the 7th leading cause of death in the United States as well as worldwide with 5.2 million deaths. Diabetes mortality is estimated at 82.4 per 100,000 [2].

T2DM is a complex metabolic disease that causes a range of insulin resistance in the target tissues or defects in insulin secretion from the pancreas [3]. Various predisposing factors for this disease have been mentioned including systemic inflammation, oxidative stress, obesity, lifestyle, diet, and the role of vitamins and minerals $[3,4]$.

During the last decade, vitamin $D$ has been linked to an increased risk of T2DM, and vitamin D supplements are thought to be an effective option in lowering the risk of T2DM. Recently, various studies have been performed to determine the effect of vitamin D supplementation on the control of diabetes in T2DM [5-7]. Previous studies suggested that receptors of Vitamin D are located within pancreatic beta cells, and adequate levels of vitamin D aids insulin sensitivity and insulin secretion [8, 9]. Moreover, vitamin D is involved in calcium regulation and through this role, it can indirectly affect insulin release from pancreatic beta cells [1].

Contradictory results have been reported in previous studies regarding the effect of vitamin $D$ on metabolic syndrome and related diseases. For combined vitamin D and calcium supplements, intervention trials have only been effective in highrisk populations (eg. those with glucose intolerance) and have reduced the incidence of T2DM [10]. However, in RCT studies, when the study was faced with low sample size, an inappropriate dose of vitamin $D$, low homogeneity of vitamin $D$ status in the 
samples, and genetic polymorphism (at least for vitamin $\mathrm{D}$ receptor), the results have shown that vitamin $D$ and calcium do not play a protective role in T2DM [11]. Vitamin D receptors are scattered in the pancreatic beta cells and immune system cells. In addition, vitamin $\mathrm{D}$ is involved in the activity of endopeptidases in calcium-dependent beta cells and can both directly induce beta cells to secrete insulin and by indirectly increasing intracellular calcium, convert calcium-dependent channels in beta cells which convert pro-insulin to insulin [12].

Hyperglycemia also causes oxidative stress by increasing protein glycation and glucose oxidation. Overproduction of reactive oxygen species and consequent depletion of the body's antioxidant defense system leads to lipid peroxidation and cell damage which then lead to macro-and microvascular complications of diabetes [13].

Due to the importance of vitamin D deficiency and macro-mineral deficiency in diabetic patients, differences in study results, differences in climatic conditions and diet in different regions, and the lack of similar studies in recent years in Shiraz city, this study aims to compare vitamin D levels, calcium, phosphorus, and oxidative conditions in diabetic and healthy patients.

\section{SUBJECTS AND METHODS}

This descriptive-analytical study was conducted in the Laboratory of Pathobiology and Genetics of Peyvand Shiraz city, Fars province, south of Iran (2020). Approval was obtained from the ethics committee of the Shiraz University of Medical Science. The procedures used in this study adhere to the tenets of the Declaration of Helsinki.

Inclusion and exclusion criteria: The population of our study was selected randomly from diabetic patients who were referred to one of the medical diagnostic labs in Shiraz city. Inclusion criteria in our investigation were diabetic patients (T2DM) who have been diagnosed for at least three years and with $\mathrm{HbA1c}$ equivalent to $6-8$, those who are taking oral medicine for the control of blood glucose (other than insulin), 20-60 years old in both genders, non-smoker, non-alcoholic, and without comorbidities. Exclusion criteria included consuming supplements in the form of minerals and vitamin $\mathrm{D}$, obesity (BMI>33), nephropathies, hepatitis, or any comorbidity that requires medical attention, those with malabsorption, infertility, oligomenorrhea, pregnancy, lactation, malignancy, use of drugs affecting bone metabolism (estrogen, calcitonin, bisphosphonates, etc.), and injection of vitamin D in the last six months.

Collection of samples: Forty healthy individuals were randomly selected from patients with no history of diabetes. The control group was matched to the patient group in terms of number, age, and sex.

The study process was explained verbally to all participants. After obtaining the informed consent of the study participants, study-related interviews were conducted with them. Those who wished to participate in this study used their blood serum to measure the amount of calcium, phosphorus, vitamin D, Malondialdehyde (MDA), and their total antioxidant capacity (TAC).

Sampling was performed after 8-12 hours of fasting from 8-11 a.m. and before taking blood glucose-lowering medications. Serum and plasma (containing the anticoagulant EDTA) were isolated to measure the target assays.

Estimation of Vitamin D, Calcium, Phosphorous, Malondialdehyde and total antioxidant capacity: Vitamin D was measured by high-pressure liquid chromatography (HPLC) according to the Galunska et al. method [14]. $0.5 \mathrm{ml}$ of the sample were mixed with $1 \mathrm{ml}$ of ethanol 96 (for 1 minute) to precipitate the protein. Then, the mixture was centrifuged for 10 
minutes at $5500 \mathrm{rpm}$ to achieve a clear supernatant. Subsequently, the clear liquid with $3 \mathrm{ml}$ of hexane was re-extracted and evaporated under a slow flow of nitrogen. The dried residue was dissolved in $0.2 \mathrm{ml}$ of methanol and HPLC analysis was carried out (Waters pump binary 1525, USA). Ten microliters of the prepared sample were injected into $25 \mathrm{~cm}$ long, 5micron diameter columns, and vitamin $\mathrm{D}$ was measured by UV-visible detector [14].

MDA content, which determined the extent of lipid peroxidation quantitatively, was assessed using a colorimetric method according to the method prepared by the Zellbio (Germany) kit. Lipid peroxidation is measured by the reaction of thiobarbituric acid with MDA.

The TAC was measured using a colorimetric approach based on Ferric Reducing Potential of Plasma (FRAP).

Calcium and phosphorus were measured by the photometric method (Pars Azmoon diagnostic kits) and with an auto-analyzer (BT-1500 Biotenica, Italy).

Statistical analysis: Demographic and laboratory information were collected after coding and entered into the SPSS 22 software. The Shapiro Wilks test was used to evaluate the normality of the data. To compare the mean of the variables in the two groups, the t-test was used if the data was normal and, if it was not normal, the Mann-Whitney test was used.

\section{RESULTS}

Demographics result: The Mean \pm SD of the participant's age was $45 \pm 9$ years (range from 20 to 60 years). There was an equal proportion of both sexes in the groups.

Vitamin $D$ result: For vitamin D levels, there was a statistically significant difference between the two groups. The level of vitamin D in the diabetic group was $29.1 \pm 2.25 \mathrm{IU} / \mathrm{mL}$ and in the control group, it was $33.5 \pm 4.5 \mathrm{IU} / \mathrm{mL}(p=0.001)$ (Table 1$).$

Calcium and phosphorus result: Comparing the level of calcium and phosphorus in diabetics and healthy people revealed no significant difference (Table 1).

\section{Malondialdehyde and total antioxidant capacity} results: There was a significant difference between the two groups in terms of MDA level $(p<0.001)$, and its value in the diabetic group was higher than the control. However, in the case of TAC, there was no significant difference between the two groups (Table 1).

Table 1. Serum levels of different variables in the two groups.

\begin{tabular}{|c|c|c|c|}
\hline Variable & $\begin{array}{c}\text { Diabetic (type 2) } \\
\text { Mean士SD }\end{array}$ & $\begin{array}{c}\text { Non-Diabetic } \\
\text { Mean士SD }\end{array}$ & P-value \\
\hline $25(\mathrm{OH})$ Vit D IU/mL & $29.1 \pm 2.25$ & $33.5 \pm 4.5$ & 0.001 \\
\hline Calcium mg/dL & $9.53 \pm 0.2$ & $9.64 \pm 0.18$ & 0.126 \\
\hline Phosphorous mg/dL & $3.62 \pm 0.37$ & $3.47 \pm 0.28$ & 0.234 \\
\hline MDA $\mu \mathrm{M}$ & $2.23 \pm 0.28$ & $1.08 \pm 0.19$ & $<0.001$ \\
\hline TAC $\mathrm{mM}$ & $1.57 \pm 0.41$ & $1.66 \pm 0.23$ & 0.473 \\
\hline
\end{tabular}

MDA Malondialdehyde; TAC: Total antioxidant capacity; Vit D: Vitamin D; SD: standard deviation 


\section{DISCUSSION}

This study aimed to appraise the level of vitamin $D$, calcium, phosphorus, and oxidative status in diabetic and non-diabetic patients.

After conducting this study, we detected that the level of vitamin $D$ in the T2DM group was significantly lower compared to the healthy group. It can be concluded that there is a role for vitamin $D$ in regulating blood sugar and improving insulin sensitivity by reducing insulin resistance [1]. This study correlates well with the study conducted by Chiu et al. among 126 healthy and glucose tolerant subjects where 47 subjects were detected to have Vitamin D levels less than 20 $\mathrm{ng} / \mathrm{ml}[8]$.

The most well-known use of vitamin $D$ is for bone protection, but research has begun to explore its role in many areas of health. It has been proven that vitamin $D$ deficiency can be a factor in developing Type 2 diabetes. It has also been demonstrated that the beta cells of the pancreas that secrete insulin contain the vitamin $D$ receptor and the enzyme alpha 1 hydroxylase [15].

The study by Osmani was conducted on 50 newly diagnosed Type 2 diabetes mellitus patients for evaluating the levels of Vitamin D in diabetic and non-diabetic patients; they found that the diabetic group had lower levels of Vitamin D compared with the control. It can be concluded that there is a role for Vitamin D in regulating blood sugar levels and improving insulin sensitivity [1].

Moreira-Lucas et al. performed a double-blind, randomized, placebo-controlled 24-week trial to assess insulin sensitivity and beta-cell function. They used 71 participants whose serum vitamin D levels were $25(\mathrm{OH}) \mathrm{D} \leq 65 \mathrm{nmol} / \mathrm{L}$ and had high fasting blood sugar and elevated glycosylated hemoglobin. For 24 weeks, once a week, cheese with and without vitamin D were given to subjects that were randomly divided into two groups of 28,000 IU of vitamin D3 (VitD; $n=$ 35) and placebo $(n=36)$. The results of their study after glucose tolerance testing in placebo and vitamin D groups showed that weekly doses of vitamin D3 in people with undesirable vitamin D levels and at risk for Type 2 diabetes could not improve oral glucose tolerance or other glycemic markers [16].

Calcium has been discovered to have an indirect effect on insulin secretion. Vitamin D can play a role in keeping extracellular calcium levels normal, thereby controlling the flow of calcium through the cell membrane. Decreased serum vitamin D levels can affect and reduce the ability of calcium to secrete insulin [1]. In our study, serum calcium and phosphorus levels did not show a statistical significant difference with the control group. In contrast to our study, Chen Hui et al. have shown that in Type 2 diabetic patients, the circulating phosphorus was lower while the circulating calcium was higher [17].

Reduced calcium absorption during vitamin D deficiency triggers the secondary release of parathyroid hormone and calcium reabsorption increases in the kidneys. This condition prevents the entry of calcium required for insulin processing in target cells and reduces insulin sensitivity by increasing intracellular calcium levels [18]. Decreased insulin sensitivity increases parathyroid hormone secretion [19]. Thus, increased parathyroid hormone due to low levels of vitamin $D$ has an unfavorable effect on insulin release from beta cells [20-21].

Another way vitamin D affects diabetes is by increasing the expression of insulin receptors and improving insulin function, as well as increasing the insulin response to glucose transport [22]. Vitamin D prevents beta-cell apoptosis and thus maintains betacell mass [23]. 
Diabetes is often correlated with elevated oxidative stress as a result of decrease of antioxidant defenses and a rise in free radical generation [24]. Free radicals attack lipids to form malondialdehyde, one of the most important markers of lipid peroxidation, following an enzymatic reaction. Increased levels of malondialdehyde indicate a disorder of the antioxidant defense system [25]. Measurement of serum malondialdehyde levels is a diagnostic indicator of lipid peroxidation and oxidative stress, which is often examined in pathological conditions caused by free radicals [26]. The results of our study were similar to the Pieme et al. study regarding the serum level of malondialdehyde, which was significantly increased in T2DM compared with the control group [26]. Li et al., in 2019, indicated that the serum level of malondialdehyde in diabetics was significantly higher when compared to the healthy group [24]. Similar reports have been made in previous studies [27-28].

TAC represents the total peroxide damage caused by low molecular weight, enzymatic, free radical particles and reflects the effect of this damage on the enzymatic and non-enzymatic antioxidant balance in the body. Increased production of ROS in body tissues and fluids has been shown to reduce TAC [29]. Derived from the findings of the current research in T2DM and healthy individuals, the TAC of these individuals did not show a statistically significant difference. This result contradicts the findings of the study by Gunawardena et al. in 2019 and Pieme et al. 2017. In their study, the difference in serum levels of total antioxidants in T2DM compared to healthy individuals was reduced and this decrease was statistically significant $[13,26]$. Also, in a study by Li et al., serum total antioxidant levels decreased in diabetics [24]. Since oxidative factors have reserves in the body, in patients with glycemic crises with insufficient reserves of TAC tissue and reduced ability to produce TAC, we can see a decrease in this oxidative factor; if the body can reproduce it, they will not observe a significant change in the level of this factor [30].

Climatic conditions affect electrolyte levels, vitamin D, and blood glucose levels. Despite access to data related to these variables, few studies have been conducted in Shiraz. Some strengths of our study were that in selecting the sample, we applied a specific age limit and a specific duration of the treatment period, and the method of measuring vitamin $D$ was done using the HPLC method. However, the use of the HPLC method and the cost of testing with this method limited the sample size.

\section{CONCLUSION}

According to our results, the average level of vitamin $D$ in diabetic patients was significantly lower than in healthy people. A lack of vitamin D can contribute to the long-term effects of diabetes, including cardiovascular disease. Due to the significant increase in MDA in T2DM patients compared to the healthy group, increased activity of MDA is a predisposing factor to the development of secondary difficulties in T2DM. By knowing more about the changes in the activity of antioxidant enzymes and oxidative factors in these patients in different stages of the disease and the factors affecting it, we can be more hopeful about increasing the effectiveness of pharmacological and nutritional interventions to reduce oxidative stress in diabetic patients. Fruits and vegetables have a protective role against chronic diseases such as cardiovascular disease, ocular and nerve diseases, stroke, cancer, diabetes, and high blood pressure. To prevent high blood pressure, stroke, cardiovascular disease, and other deficiencies related to micronutrients, it is recommended to consume at 
least 400-500 grams of fruits and vegetables daily, because insufficient intake of these substances causes non-communicable nutritional disorders. The protective effect of fruits and vegetables is mostly attributed to their antioxidant compounds that neutralize natural radicals such as vitamins $E$ and $C, E$ (alpha-tocopherol), alpha and beta carotene, and glutathione. Pigmented fruits and vegetables that contain beta and alpha carotenoids, lutein, lycopene, zeaxanthin, astaxanthin, $\beta$-cryptoxanthin act as a precursor of vitamin $A$ in the human body and in addition to compensating for vitamin deficiency in the body, play effective roles as anti-oxidants, suppressing inflammatory responses, modulating cell signaling, and inducing apoptosis. Also, they can play a protective role in cardiovascular disease, cancer, gastrointestinal disorders, and metabolic diseases [31]. Therefore, diabetic patients may need more antioxidants, and taking supplements such as vitamins $\mathrm{D}$ and $\mathrm{C}$ can have a critical role in strengthening the system of antioxidant protection and improving the lives of diabetic patients.

\section{REFERENCES}

1. Osmani D, Haseena S: A possible correlation between low serum vitamin-D levels and type 2 diabetes mellitus. International Journal of Advanced Biochemistry Research 2020, 4:06-11.

2. Glovaci D, Fan W, Wong ND: Epidemiology of Diabetes Mellitus and Cardiovascular Disease. Current Cardiology Reports 2019, 21(4):21.

3. Magkos F, Yannakoulia M, Chan JL, Mantzoros CS: Management of the Metabolic Syndrome and Type 2 Diabetes Through Lifestyle Modification. Annual Review of Nutrition 2009, 29(1):223-256.

4. Kern HJ, Mitmesser SH: Role of nutrients in metabolic syndrome: a 2017 update. Nutrition and Dietary Supplements 2018, 10:13-26.

5. Bhatt SP, Misra A, Pandey RM, Upadhyay AD, Gulati S, Singh N: Vitamin D Supplementation in Overweight/obese Asian Indian Women with Prediabetes Reduces Glycemic Measures and Truncal Subcutaneous Fat: A 78 Weeks Randomized Placebo-Controlled Trial (PREVENT-WIN Trial). Scientific Reports 2020, 10(1):220.

6. Park SK, Garland CF, Gorham ED, BuDoff L, BarrettConnor E: Plasma 25-hydroxyvitamin D concentration and risk of type 2 diabetes and pre-diabetes: 12-year cohort study. PloS one 2018, 13(4):e0193070.
Abbreviations: MDA: malondialdehyde, TAC: total antioxidant capacity, DM: Diabetes mellitus, BMI: body mass index, EDTA: Ethylenediaminetetraacetic acid, HPLC: high-pressure liquid chromatography, SD: Standard deviation, Vit D: Vitamin D.

Authors' contribution: Study concept and design: Maryam Barghi, Amir Sadeghipoor Ranjbar; Analysis and interpretation of data: Homa Moazen, and Narges Eskandari-Roozbahani; Drafting of the manuscript: Maryam Barghi, Amir Sadeghipoor Ranjbar, Narges Eskandari-Roozbahani; Critical revision of the manuscript for important intellectual content: Narges Eskandari-Roozbahani, Maryam Barghi; Statistical analysis: Homa Moazen, and Narges Eskandari-Roozbahani

Funding: The author(s) received no financial support for the research, authorship, and/or publication of this article.

Conflict of interest: The authors declare that there is no conflict of interest

7. Xia J, Song Y: Vitamin D status during pregnancy and the risk of gestational diabetes mellitus: A longitudinal study in a multiracial cohort. 2019, 21(8):1895-1905.

8. Chiu KC, Chuang LM, Yoon C: The vitamin D receptor polymorphism in the translation initiation codon is a risk factor for insulin resistance in glucose tolerant Caucasians. BMC Med Genet 2001, 2:2-2.

9. Scragg R, Sowers M, Bell C: Serum 25-hydroxyvitamin D, diabetes, and ethnicity in the Third National Health and Nutrition Examination Survey. Diabetes care 2004,27(12):2813-2818.

10. 1Pittas AG, Lau J, Hu FB, Dawson-Hughes B: The role of vitamin $D$ and calcium in type 2 diabetes. A systematic review and meta-analysis. The Journal of Clinical Endocrinology and Metabolism 2007, 92(6):2017-2029.

11. Garbossa SG, Folli F: Vitamin D, sub-inflammation, and insulin resistance. A window on a potential role for the interaction between bone and glucose metabolism. Reviews in Endocrine and Metabolic Disorders 2017, 18(2):243-258.

12. Mitri J, Pittas AG: Vitamin D and diabetes. Endocrinology and Metabolism Clinics 2014, 43(1):205- 232.

13. Gunawardena HP, Silva R, Sivakanesan R, Ranasinghe $P$, Katulanda P: Poor Glycaemic Control Is Associated with Increased Lipid Peroxidation and Glutathione Peroxidase Activity in Type 2 Diabetes Patients. Oxidative 
Medicine and Cellular Longevity 2019, 2019:9471697.

14. Galunska B, Gerova D, Boncheva M, Svinarov D: HPLC method for measuring the circulating levels of 25hydroxy vitamin D: validation and comparison with ID LC/MS/MS and immunoassay. Integr Food Nutr Metab 2014, 1(2):119-123.

15. Bland R, Markovic D, Hills CE, Hughes SV, Chan SL, Squires $\mathrm{PE}$, Hewison M: Expression of 25-hydroxyvitamin D3-1alpha-hydroxylase in pancreatic islets. Steroid Biochem Mol Biol 2004, 89-90(1-5):121-125.

16. Moreira-Lucas TS, Duncan AM, Rabasa-Lhoret R, Vieth $R$, Gibbs AL, Badawi A, Wolever TM: Effect of vitamin D supplementation on oral glucose tolerance in ndividuals with low vitamin $D$ status and increased risk for developing type 2 diabetes (EVIDENCE): A double-blind, randomized, placebo-controlled clinical trial. Diabetes, obesity and metabolism 2017, 19(1):133-141.

17. Chen H, Li X, Yue R, Ren X, Zhang X, Ni A: The effects of diabetes mellitus and diabetic nephropathy on bone and mineral metabolism in T2DM patients. Diabetes research and clinical practice 2013, 100(2):272-276.

18. Stivelman $E$, Retnakaran R: Role of vitamin $D$ in the pathophysiology and treatment of type 2 diabetes. Current Diabetes Reviews 2012, 8(1):42-47.

19. Alvarez JA, Ashraf A: Role of vitamin D in insulin secretion and insulin sensitivity for glucose homeostasis. International journal of endocrinology 2010, 2010.

20. George P, Pearson E, Witham M: Effect of vitamin D supplementation on glycaemic control and insulin resistance: a systematic review and meta-analysis. Diabetic Medicine 2012, 29(8):e142-e150.

21. Onalan E, Gozel N: Prevalence of vitamin D deficiency in patients with type 2 diabetes and its relationship with glycemic control. Annals of Medical Research 2019, 26(2):222-225

22. Zipitis CS, Akobeng AK: Vitamin D supplementation in early childhood and risk of type 1 diabetes: a systematic review and meta-analysis. Archives of disease in childhood 2008, 93(6):512-517.

23. Bourlon PM, Billaudel B, Faure-Dussert A: Influence of vitamin D3 deficiency and 1,25 dihydroxy vitamin D3 on de novo insulin biosynthesis in the islets of the rat endocrine pancreas. The Journal of endocrinology 1999, 160(1):87-95.

24. Li J, Shen X: Oxidative stress and adipokine levels were significantly correlated in diabetic patients with hyperglycemic crises. 2019, 11:13.

25. Niedowicz DM, Daleke DL: The role of oxidative stress in diabetic complications. Cell biochemistry and biophysics 2005, 43(2):289-330.

26. Pieme CA, Tatangmo JA, Simo G, Biapa Nya PC, Ama Moor VJ, Moukette Moukette B, Tankeu Nzufo F, Njinkio Nono BL, Sobngwi E: Relationship between hyperglycemia, antioxidant capacity and some enzymatic and nonenzymatic antioxidants in African patients with type 2 diabetes. BMC research notes 2017, 10(1):141.

27. Amiri Kojuri N, Esmaeili AH: Factors evaluation of oxidantantioxidant malondialdehyde, plasma total antioxidant and vitamin C in type 1 diabetes patients compared with healthy people. Razi Journal of Medical Sciences 2019, 26(5):79-86.

28. Okoduwa S, Umar A, Ibrahim S, Bello F: Relationship of oxidative stress with type 2 diabetes and hypertension. J Diabetol 2013, 1(2).

29. Li J, Shen X: Oxidative stress and adipokine levels were significantly correlated in diabetic patients with hyperglycemic crises. Diabetology and metabolic syndrome 2019, 11:13-13.
30. Meng X, Gong C, Cao B, Peng X, Wu D, Gu Y, Wei L, Liang $X$, Liu M, Li W: Glucose fluctuations in association with oxidative stress among children with T1DM: comparison of different phases. The Journal of Clinical Endocrinology and Metabolism 2015, 100(5):1828-1836.

31. Jideani AIO, Silungwe $H$, Takalani T, Omolola AO, Udeh HO, Anyasi TA: Antioxidant-rich natural fruit and vegetable products and human health. International Journal of Food Properties 2021, 24(1):41-67. 